\title{
Comprehensive Analysis of the Components of Walnut Kernel (Juglans regia L.) in China
}

\author{
Shuxiang Geng, ${ }^{1}$ Delu Ning $\mathbb{D}^{1},{ }^{1}$ Ting Ma, ${ }^{1}$ Haiyun Chen, ${ }^{1}$ Yinzhi Zhang, ${ }^{2}$ and Xiulan Sun ${ }^{2}$ \\ ${ }^{1}$ Yunnan Academy of Forestry, Kunming, Yunnan 650201, China \\ ${ }^{2}$ State Key Laboratory of Food Science and Technology, School of Food Science, \\ Synergetic Innovation Center of Food Safety and Nutrition, Jiangnan University, Wuxi, Jiangsu 214122, China
}

Correspondence should be addressed to Delu Ning; ningdelu@163.com

Received 22 March 2020; Revised 15 February 2021; Accepted 22 March 2021; Published 5 April 2021

Academic Editor: Fuguo Liu

Copyright (c) 2021 Shuxiang Geng et al. This is an open access article distributed under the Creative Commons Attribution License, which permits unrestricted use, distribution, and reproduction in any medium, provided the original work is properly cited.

\begin{abstract}
The contents of main components in 45 walnut kernels from 5 walnut-planting provinces in China (Yunnan, Shaanxi, Shandong, Hebei, and Sichuan) were determined using colorimetry, high-performance liquid chromatography, and gas chromatography, including flavonoids, vitamin E, trace elements, fatty acids, and amino acids. The levels of flavonoids and vitamin E were higher in walnuts from Yunnan than in walnuts from the other four provinces. The levels of zinc and iron were relatively higher in walnuts from Shandong, and the calcium content in walnuts from Yunnan was much lower. No obvious difference was found in crude fat concentration for nuts from the five provinces, but differences were observed in the crude protein content. Oleic acid was the predominant unsaturated fatty acids in all walnut species; the monounsaturated fatty acid content was the highest in walnuts from Yunnan. The data obtained here provided insight into differences in walnuts resulting from different growing environments and germplasm genetic traits.
\end{abstract}

\section{Introduction}

Walnut (Juglans regia) is considered as a high-quality food with human health benefits because walnut kernels are rich in proteins, unsaturated fatty acids, phospholipids, vitamins, minerals, essential fatty acids, and other nutrients. Walnut has important medicinal value and health function in preventing and alleviating cardiovascular disease, diabetes, and obesity [1].

The common walnut (Juglans regia L.) is an economically important species cultivated worldwide for both highquality wood and nuts. Walnut cultivation has a long history and is widely cultivated in most areas of the Eurasian continent [2]. However, the relative scarcity of wide-scale molecular phylogeographic studies of walnut makes it difficult to accurately determine the native geographic range. China is a major center of walnut genetic diversity, serving as a germplasm source for walnut breeding efforts $[3,4]$. Starting in the Western Han Dynasty (206 BCE to $9 \mathrm{CE}$ ), walnuts were cultivated by selecting seedlings from geographically distinct natural populations and then spread by trade and military conquest $[2,5]$. Many classical studies on the origin of crops in the world explored the original crops in China and proposed that China was one of the largest original centers of agriculture and cultivated plants $[6,7]$.

In the walnut genus (Juglans), three species originated in China (Juglans mandshurica Max., Juglans Hopeiensis $\mathrm{Hu}$, and Juglans cathayensis Dode); and walnut Juglans regia L. originated outside of China [8]. Walnut resources are widely distributed in China, in Yunnan, Hebei, Shandong, Shandong, Liaoning, Sichuan, Shaanxi, and Tibet [9, 10]. Many studies reported significant differences in the appearance, value, and nutritional composition of walnuts from different production areas [11]. These differences may result from variation in the growing environment or genetic variations. A better understanding of the origin, the genetic diversity, and the structure of wild populations of common walnut in China is needed to address the source of these differences. 
The sampled sites in this study were located in (1) Yunnan, where J. regia and J. sigillata are sympatric, and in regions containing J. regia and other Juglans species native to China; (2) Qinling-Daba Mountains, including Shaanxi and Sichuan, which are regions known to support high levels of genetic diversity; and (3) J. mandshurica Maxim., which belongs to the Juglans genus of the Juglandaceae family and primarily grows in northeastern China along the Yellow River basin, near Hebei and Shandong [12] (Figure 1). Moreover, the five regions have different geomorphological and climatic types, among which Yunnan is mountainous plateau terrain and has subtropical monsoon climate. Sichuan is basin terrain and has subtropical humid monsoon climate, Shaanxi is loess plateau terrain and has north warm temperate zone climate, and Hebei and Shandong are mainly plain terrain with a temperate monsoon climate. Obviously, their temperate, annual precipitation, and daylight are different. Thus, this study aimed to compare the main components of walnut kernels from different walnut-producing provinces in China (Yunnan, Shaanxi, Shandong, Hebei, and Sichuan); the data were used to estimate the degree of main component differentiation for walnuts in China.

\section{Materials and Methods}

2.1. Chemicals and Reagents. All chemicals and reagents were of analytical grade or of high-performance liquid chromatography (HPLC) (99.9\%)/gas chromatography (GC) (>99.5\%) grade from Beyotime Co., Ltd (Nantong, China) and Tianjin Chemical Company, Ltd. (Tianjin, China). Standards were from Sigma Chemical Co. (St. Louis, MO, USA), unless indicated otherwise.

2.2. Walnut Samples. A total of 45 walnut varieties were obtained on their pick-up time (between July and October, 2017) from Yunnan, Shaanxi, Hebei, and Shandong provinces in China. 33 varieties were derived from Yunnan-Kweichow plateau including Yunxin series (nos. 64, 303, 36, 4-3, 301, and 306), Yangpao series (Fengqing, Lijiang-1, Jingdong, Shuangjiang, Dali-Yongping, Lincang, Yangbi, Lijiang-2, Diqing, Malin-Machang-1734, Yangbi-1902, Yangpao-21662, Guangming-2300, and Yangpao-2563), and other 15 main varieties (Baoshan-Xixiang, Zijin, Dayao-Santai, Yongpao-1, Huaning-Dashake, Huaning-Dabaike, Yunnan-Dabaike, Yunnan-Dashake, Niangqing-Ziren, Yunnan-Dayuan, Yunnan-Xiaoyuan, Niangqing-Bairen, and Yong-11). 5 varieties were from Shaanxi (Liaohui, Xiangling, Zhonglin-5, Liaohe-4, and Xifu-2). 3 varieties were from Hebei (Liao-1, Zanmei, and Qingxiang), and 2 varieties were from Shandong (Jinlong-2 and Luguang) and Sichuan (Sichuan-Yuanyuanzao and Zhonglin-1), respectively.

In short, fresh walnuts were broken, and the shells were taken out. The walnuts were dried in a drying oven at a constant temperature of $45^{\circ} \mathrm{C}$, until the moisture content was less than 10\%; the moisture content was recorded. All samples were then grounded with a grinder, sealed, and stored away from light at $-20^{\circ} \mathrm{C}$.
2.3. Lipid Extraction. Lipids were extracted from 45 samples of walnut kernels by Soxhlet extraction method with $n$ hexane as the solvent [13]. For each extraction, $3.0 \mathrm{~g}$ dried walnut kernels were packed in a filter paper and inserted into the Soxhlet apparatus. The organic solvent $(200 \mathrm{~mL})$ was added, and then the system was heated until the evaporation of the solvent. After $6 \mathrm{~h}$ reflux extraction, the major solvent was recycled, and the material was then dried for $2 \mathrm{~h}$ in a drum wind drying oven at $60^{\circ} \mathrm{C}$. After cooling in a desiccator for $1 \mathrm{~h}$, the flask was weighed and adjusted to a constant value.

\subsection{Fatty Acid Analysis}

2.4.1. Fatty Acid Derivatization. Fatty acids in the oil extracts were measured as follows. First, $100 \mu \mathrm{L}$ of the oil extracts from the walnut kernels was mixed with $4 \mathrm{~mL}$ of $n$ hexane and then incubated for $15 \mathrm{~min}$ at room temperature. The organic solution was then combined with $2.0 \mathrm{~mL}$ of $4 \%$ potassium hydroxide and vortexed for $30 \mathrm{~s}$. Next, equal volume of distilled water was added to wash the alkali in the organic phase, and the mixture was allowed to stand for $2 \mathrm{~min}$. The upper $n$-hexane layer was then transferred to a new tube and then was washed with distilled water three times. Finally, about $0.15 \mathrm{~mL}$ of the upper hexane layer was transferred to a glass vial and stored at $-20^{\circ} \mathrm{C}$ prior to analysis by GC-mass spectrometry (GC-MS) [14, 15].

2.4.2. GC-MS Conditions. The fatty acid methyl esters (FAME) in each sample were identified using a GC-MS-QP2010 Ultra (Shimadzu Instruments, Japan). A DB wax column (30 m length $\times 0.25 \mathrm{~mm}$ internal diameter $\times 0.25 \mu \mathrm{m}$ film thickness; USA) was used; and helium was used as a carrier gas $(1.5 \mathrm{~mL} /$ $\mathrm{min})$. Samples were injected in the split mode $(0.3 \mu \mathrm{L}$, split ratio 30) with an initial temperature of $80^{\circ} \mathrm{C}$ for $2 \mathrm{~min}$, then ramped at $10^{\circ} \mathrm{C} / \mathrm{min}$ to $160^{\circ} \mathrm{C}$, held for $5 \mathrm{~min}$, increased to $250^{\circ} \mathrm{C}$ at a rate of $5^{\circ} \mathrm{C} / \mathrm{min}$, and then held for $8 \mathrm{~min}$. Mass spectra were acquired over 35-500 amu at 10 scans per second. The injector and detector temperatures were maintained at $250^{\circ} \mathrm{C}$ and $300^{\circ} \mathrm{C}$, respectively [16]. The identity and area of the FAME peaks in the samples were identified by matching the retention time (s) with the FAME standards using GC-MS solution software (Shimadzu Scientific Instruments) as well as by matching the mass spectra with the NIST14 library.

2.5. Total Protein Determination. The protein contents of the walnut kernels were determined by the Kjeldahl method using Kjeltec 8400 with an AOAC system (Foss, USA) [17]. The measured nitrogen content was converted into total protein content by applying a conversion factor of 6.25. The measurements were performed in triplicate, and the average values were presented.

2.6. Amino Acid Analysis. For amino acid analysis, $100 \mathrm{mg}$ of dried walnut kernels powder and $8 \mathrm{~mL}$ of $6.0 \mathrm{~mol} / \mathrm{L} \mathrm{HCl}$ were mixed in a sealed tube, and then hydrolysis was carried out at $120^{\circ} \mathrm{C}$ for $22 \mathrm{~h}$ after nitrogen filling for $3 \mathrm{~min}$. After 


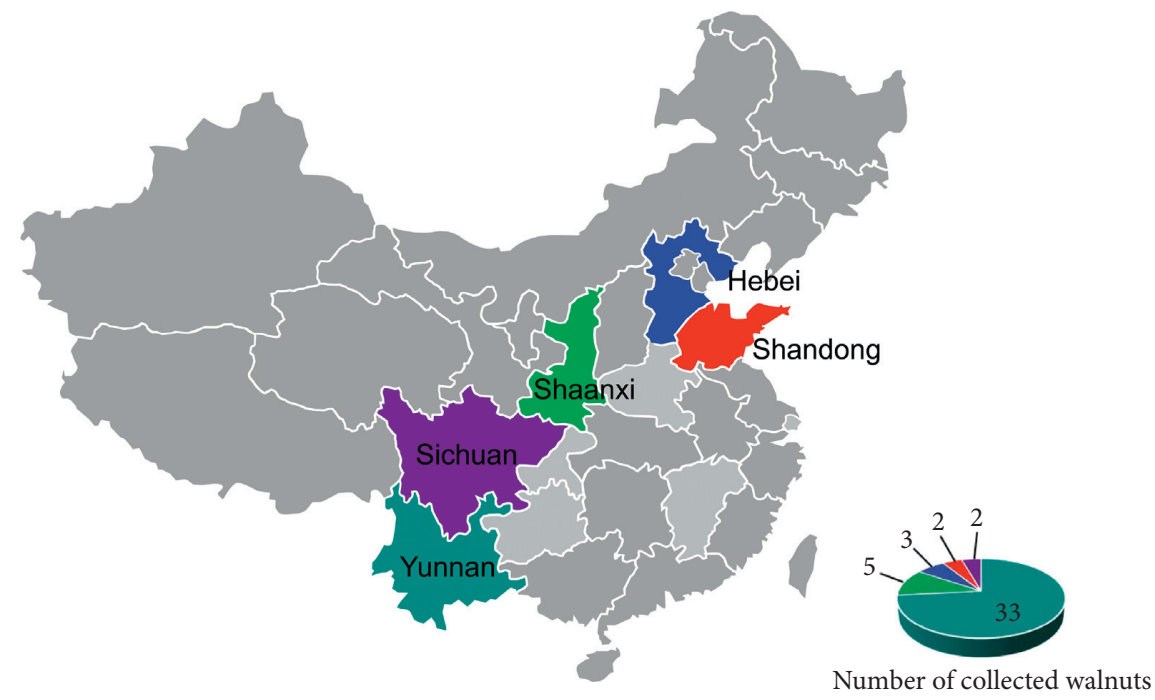

Figure 1: The walnut kernel samples collection from the main Chinese provinces for planting walnut. The inset pie chart displays the number of walnut types from the main five walnut-planting provinces.

cooling, $4.8 \mathrm{~mL}$ of $10 \mathrm{~mol} / \mathrm{L} \mathrm{NaOH}$ was added to neutralize the sample for acid hydrolysis and diluted with deionized water to $25 \mathrm{~mL}$. Next, $1 \mathrm{~mL}$ of the sample was centrifuged at $10,000 \mathrm{rpm}$ for $10 \mathrm{~min}$ and filtered through a $0.45 \mu \mathrm{m}$ Millipore filter.

Subsequently, a chromatographic analysis was performed using a HPLC system equipped with a photodiode array detector (PDA) from Agilent (Agilent 1260, USA) [18] s. A sample of $10 \mu \mathrm{L}$ filtrate was applied to the HPLC, using a column packed with a custom ion-exchange resin $(4.6 \mathrm{~mm} \times 100 \mathrm{~mm}$, particle size $7 \mu \mathrm{m})$, column temperature of $35^{\circ} \mathrm{C}$, sample injection rate of $0.8 \mathrm{~mL} / \mathrm{min}$, and monitoring at $570 \mathrm{~nm}$ and $440 \mathrm{~nm}$. The amino acid concentrations were expressed in $\mathrm{g} / 100 \mathrm{~g}$ dry weight.

2.7. Total Flavonoid Detection. The amount of total flavonoids in the walnut kernels was determined by ultraviolet or visible spectrophotometry, using rutin as the contrast sample and $\mathrm{Al}\left(\mathrm{NO}_{3}\right)_{3}$ as the revealing agent [19]. Further, $0.5 \mathrm{~g}$ of each sample was mixed with $15 \mathrm{~mL}$ of methanol, ultrasonically extracted for $30 \mathrm{~min}$, cooled rapidly to room temperature, and then diluted with methanol to $30 \mathrm{~mL}$. Next, $1.0 \mathrm{~mL}$ extracts were combined with $1 \mathrm{~mL}$ of $0.5 \%$ $\mathrm{NaNO}_{2}$ solution. After resting for $5 \mathrm{~min}, 1 \mathrm{~mL}$ of $10 \%$ $\mathrm{Al}\left(\mathrm{NO}_{3}\right)_{3}$ solution was added and held for $6 \mathrm{~min}$ before adding $5 \mathrm{~mL}$ of $4 \% \mathrm{NaOH}$ solution. The sample was then incubated in a $37^{\circ} \mathrm{C}$ water bath for 10 min after dilution with $30 \%$ methanol to $25 \mathrm{~mL}$. A detection wavelength of $510 \mathrm{~nm}$ was used for measurement. Rutin was solubilized with methanol and diluted to appropriate concentrations to generate the standard curve following the aforementioned extraction steps.

2.8. Vitamin E Analysis. Vitamin E concentrations in walnut kernels were determined by reversed-phase HPLC, using fluorescence and visible detection [20]. Sample processing of the 45 walnut samples was consistent with the fatty acid derivatization described earlier. For the mobile phase, $n$ hexane was used with a flow rate of $1.0 \mathrm{~mL} / \mathrm{min}$. The injection volume was $10 \mu \mathrm{L}$, and the temperature of the column was $30^{\circ} \mathrm{C}$. The excitation and emission wavelengths were $294 \mathrm{~nm}$ and $328 \mathrm{~nm}$, respectively. Quantification was performed by comparison against the $\gamma$-tocopherol standard curve at appropriate concentrations, and the results were expressed as $\mathrm{mg} / \mathrm{g}$ of walnuts.

2.9. Trace Elements. For measuring the levels of trace elements in the walnut kernel samples, $0.5 \mathrm{~g}$ powdered walnut kernels from the 45 walnut samples were mixed with $6.0 \mathrm{~mL}$ of nitric acid in the digestion tank and predigested at $120^{\circ} \mathrm{C}$ for $15 \mathrm{~min}$. Then, $1 \mathrm{~mL}$ of $30 \% \mathrm{H}_{2} \mathrm{O}_{2}$ was added, and the tank was put into the digestion system for further digestion. The operating conditions were (1) $130^{\circ} \mathrm{C}, 1,013 \mathrm{kPa}, 5 \mathrm{~min}$; (2) $150^{\circ} \mathrm{C}, 1,520 \mathrm{kPa}, 5 \mathrm{~min}$; and $(3) 180^{\circ} \mathrm{C}, 2,230 \mathrm{kPa}, 5 \mathrm{~min}$. Next, $15 \mathrm{~mL}$ of distilled water was added and heated to remove the acid. The samples were then washed with $2 \%$ nitric acid several times before the sample volume was adjusted to $25 \mathrm{~mL}$ by adding distilled water. The simultaneous analysis of $\mathrm{Zn}, \mathrm{Fe}$, and $\mathrm{Ca}$ was performed using a Perkin Elmer 2380 Model FAAS [21]. The absorption data were obtained as the averages of three measurements and were evaluated based on the least-square regressed calibration graphs.

2.10. Statistical Analysis. The results were expressed as mean \pm standard deviation (SD) of at least three replicates. The data were statistically analyzed using the Statistical Package for Social Sciences (IBM SPSS Statistics) software, version 22.0. STATA 12 was used to plot the graphs. Data were analyzed by analysis of variance (ANOVA) followed by Scheffe's post hoc test and principal component analysis (PCA) method $(p<0.05)$. 


\section{Results and Discussion}

3.1. Flavonoids and Vitamin E. Flavonoids are considered important components in the human diet, although they are generally considered nonnutrients. In the common walnut (Juglans regia L.), flavonoids are considered the major phenolic compounds [22]. Flavonoid concentrations in 45 samples of walnut kernels from the main 5 walnut-producing provinces in China were detected, as shown in Figure 2(a). The flavonoid concentrations were less than $5 \%$ for walnuts species cultivated in the Qinling-Daba Mountains, including Shaanxi and Sichuan. The flavonoid concentrations of walnuts were also lower than 5\%, in northeastern China along the Yellow River basin, including Hebei and Shandong, than in Shaanxi and Sichuan. The flavonoid concentrations in walnuts from Yunnan were higher than those from the other four provinces, with an average concentration around $6.4 \%$.

The major dietary vitamin E sources are vegetable oils, nuts, cereals, green vegetables, and fruits. The consumption of walnuts is a healthy choice due to the high vitamin E content. The vitamin E composition is influenced by environmental factors, besides genetic factors [23]. The concentration of vitamin $\mathrm{E}$ was measured in the walnut kernels from the five main walnut-producing provinces in China, as shown in Figure 2(b). The average vitamin E concentration in walnuts cultivated in the Qinling-Daba Mountains, including Shaanxi and Sichuan, and in Hebei and Shandong in northeastern China along the Yellow River basin, was lower than $0.9 \mathrm{mg} / \mathrm{g}$. The vitamin $\mathrm{E}$ concentration was higher in walnuts from Yunnan than in walnuts from the other four provinces, with an average concentration of $1.09 \pm 0.17 \mathrm{mg} / \mathrm{g}$. Also, the greater genetic diversity of walnuts from Yunnan led to a larger range of vitamin E concentrations (shown in Figure 2(b)).

3.2. Trace Elements. The study next compared the levels of minerals and trace elements in the walnuts from the 5 walnut-producing provinces to establish the influence of variety and origin. Zinc is one of the most important trace elements for humans, as it is a cofactor for a number of enzymes that interact with DNA and RNA, such as zinc finger proteins and RNA polymerase [24]. Zinc is involved in regulating a wide variety of physiological responses, such as growth, immunity, neurons, and sensory function $[25,26]$. As shown in Figure 3(a), the zinc content was analyzed in the 45 walnut kernel samples. The results showed the highest zinc content in walnuts from Shandong $(0.06 \pm 0.01 \mathrm{mg} / \mathrm{g})$ and the lowest content in walnuts from Hebei $(0.03 \pm 0.01 \mathrm{mg} / \mathrm{g})$. The content of zinc in walnuts from Yunnan was almost of the same level as that in the walnuts from Shaanxi and Sichuan but a bit lower.

Iron deficiency anemia is common in infancy and childhood as well as during pregnancy [27]. Adequate iron content enhances hematopoietic function and improves human immunity. The iron content is relatively higher in walnuts than in other nuts $[28,29]$. Therefore, the iron levels were measured in the 45 walnut kernel samples. As shown in Figure 3(b), the iron content in walnuts from Hebei was $0.04 \pm 0.01 \mathrm{mg} / \mathrm{g}$, which was the lowest content for the 5 sample regions. The iron content in walnuts from Shandong was relatively higher than that in walnuts from Shaanxi, Sichuan, and Yunnan; and the iron contents in walnuts from these three provinces were very similar. In addition, two walnut species (Dali-Yongping and Yongpao-1) from Yunnan showed a significantly higher level of iron $(0.19 \pm 0.03 \mathrm{mg} / \mathrm{g}$ and $0.17 \pm 0.05 \mathrm{mg} / \mathrm{g}$, respectively), which might be a species-specific property or reflect a difference in the growing environment.

Calcium not only promotes bone health but also is required to maintain the normal functioning of the brain and heart $[30,31]$. Calcium participates in cellular regulation in the human body and is one of the major minerals in walnuts [32]. As shown in Figure 3(c), little variations were found in the calcium concentration of the walnut samples from the five provinces, despite a greater range of calcium content among the samples from Yunnan $(0.71 \mathrm{mg} / \mathrm{g}-0.25 \mathrm{mg} / \mathrm{g})$.

3.3. Crude Fat and Crude Protein. Fat typically provides essential fatty acids and more than half of the body's energy needs. It makes up around $60 \%-70 \%$ of the nutrient contents in walnuts, and most of this is high-quality fat [33]. Next, the amount of crude fat was measured in the 45 walnut kernel samples. As shown in Figure 4(a), no noticeable difference was found in the crude fat content in walnut kernels from the five provinces. The crude fat content in walnuts from Yunnan $(60.43 \pm 5.55 \%)$ was a little higher than that in walnuts from Shandong $(55.67 \pm 3.67 \%)$ and Shaanxi $(56.66 \pm 4.77 \%)$. Among the different types of walnuts in Yunnan province, Niangqing-Ziren had the lowest content crude fat (45\%) and Yangbi-Yangpao had the highest content crude fat $(69.99 \%)$.

Protein is a basic material in the human body with many vital roles in all kinds of metabolic activities. Walnut kernels are a good source of proteins, with immunoregulatory, antioxidant, and antitumor effects [34]. This study analyzed the crude protein content in the 45 walnut samples and found considerable differences in walnuts from different growing regions. As shown in Figure 4(b), the average content of crude protein was up to $15.0 \%$ in walnut kernels from Hebei and Shandong provinces, which was higher than that in most walnut types from other provinces. In the Qinling-Daba Mountains, the content of crude protein in walnuts from Shaanxi and Sichuan was a little bit lower $(14.75 \pm 0.48 \%)$. In 33 walnut types from Yunnan, the average content of crude protein was relatively lower $(13.8 \pm 1.22 \%)$, with a wide range from $10.97 \%$ to $16.14 \%$.

3.4. Total Fatty Acids. Walnut varieties from different production areas may have the same fatty acid composition, yet the relative content of individual components may be significantly different. Linoleic acid, oleic acid, linolenic acid, stearic acid, and palmitic acid are the major fatty acids found in walnuts [35]. Thus, the contents of saturated fatty acids (SFAs), polyunsaturated fatty acids (PFAs), monounsaturated fatty acids (MFAs), stearic acid, oleic acid, linoleic acid, and linolenic acid in the 45 walnut kernel samples were measured, as shown in Figure 5(a). Overall, the SFA contents in walnuts 


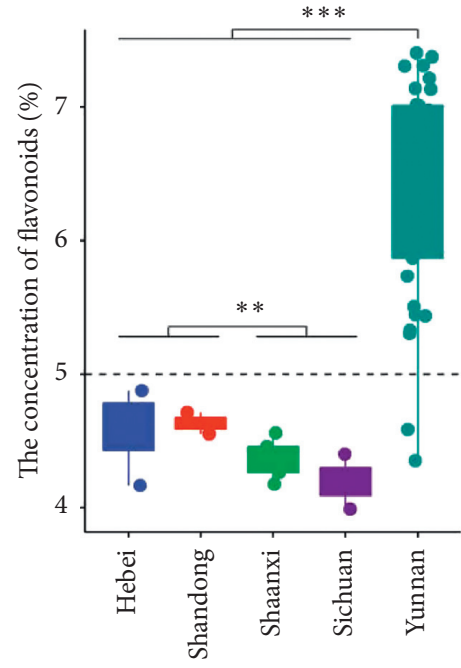

Five provinces in China

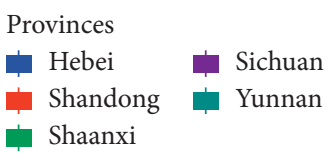

(a)

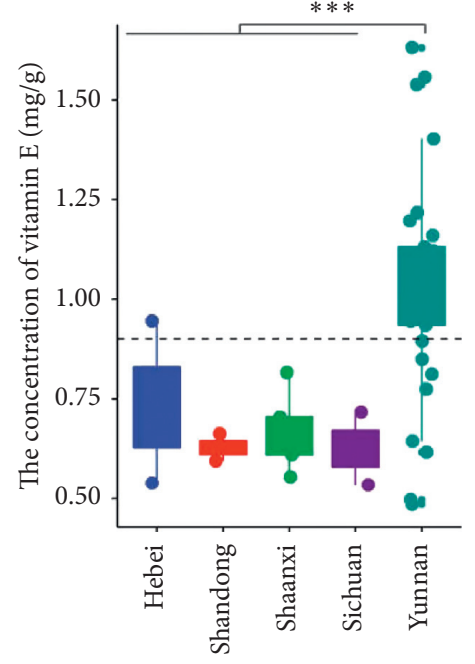

Five provinces in China

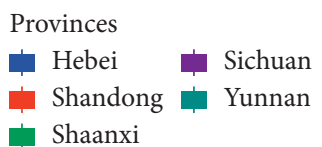

(b)

Figure 2: (a) The percentage of flavonoids and (b) the concentration of vitamin $\mathrm{E}$ in 45 different types of walnut kernels from the main five walnut-planting provinces in China. Dunnett's multiple-comparison tests were implemented for statistical analysis. Raw $p$ value was labeled, ${ }^{*} p<0.05,{ }^{* *} p<0.01$, and ${ }^{* * *} p<0.001$.

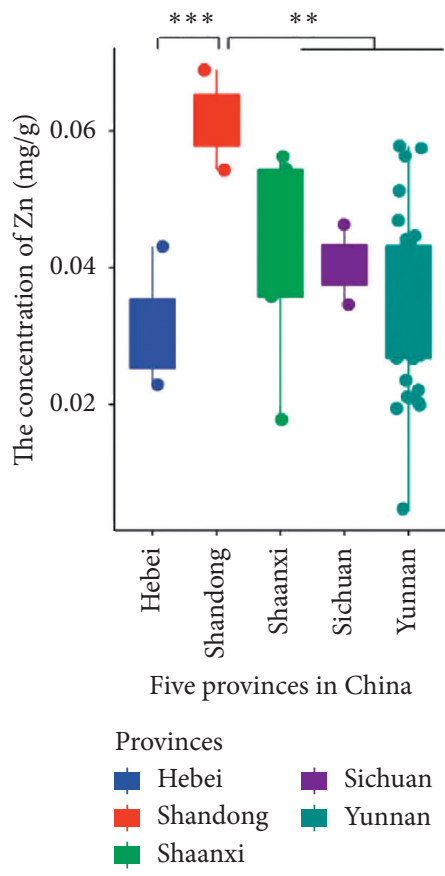

(a)

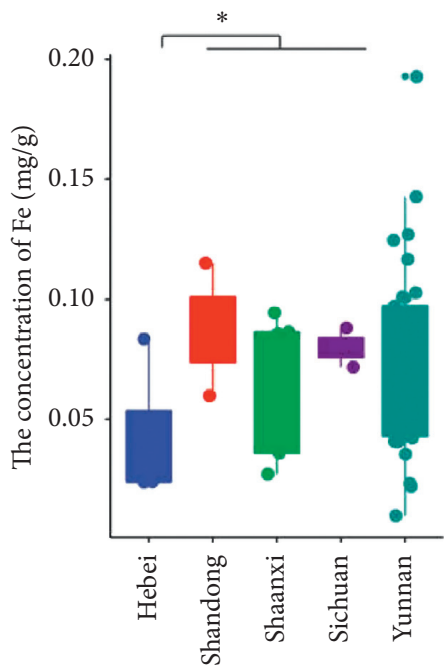

Five provinces in China

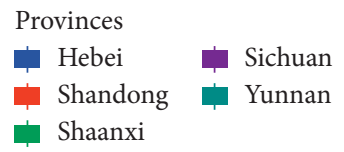

(b)

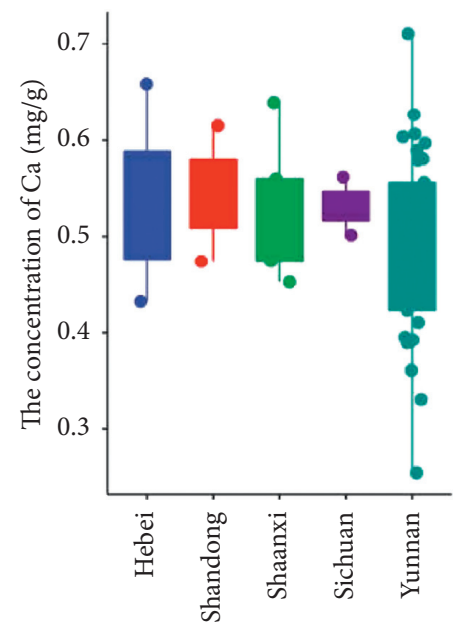

Five provinces in China

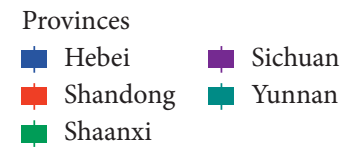

(c)

FIgURe 3: The trace elements (a) zinc, (b) iron, and (c) calcium in 45 different types of walnut kernels from the main five walnut-planting provinces in China. Dunnett's multiple-comparison tests were implemented for statistical analysis. Raw $p$ value was labeled, ${ }^{*} p<0.05$, ${ }^{* *} p<0.01$, and ${ }^{* * *} p<0.001$. 


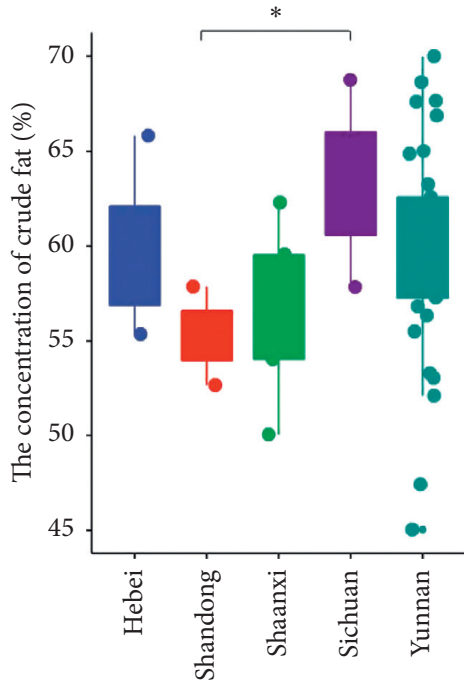

Five provinces in China Provinces

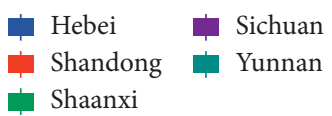

(a)

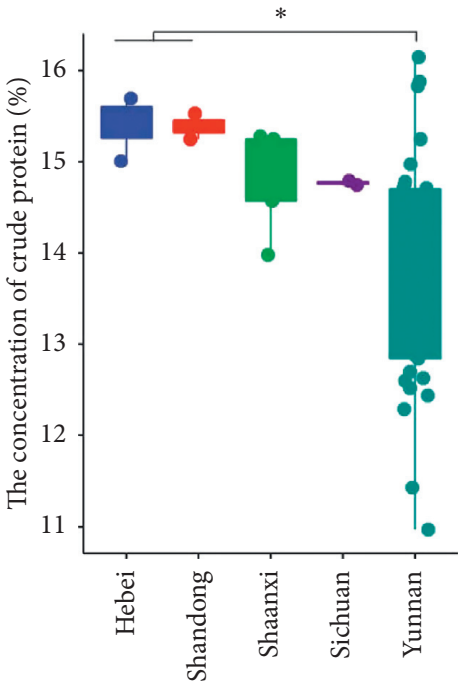

Five provinces in China

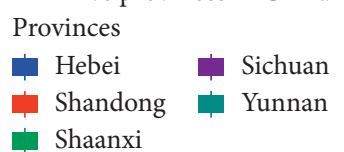

(b)

Figure 4: . The (a) crude fat and (b) crude protein in 45 different types of walnut kernels from the main five walnut-planting provinces in China. Dunnett's multiple-comparison tests were implemented for statistical analysis. Raw $p$ value was labeled, ${ }^{*} p<0.1$ and ${ }^{* *} p<0.05$.

from Hebei, Shaanxi, and Sichuan were pretty similar (the average contents were $8.79 \pm 0.44 \%, 8.24 \pm 0.80 \%$, and $8.63 \pm 1.35 \%$, respectively). The average content of SFA was $7.53 \pm 0.55 \%$ in Yunnan walnut kernels, which was significantly lower than the levels in walnuts from the other four provinces. This might be related to environmental factors or walnut varieties.

Nuts are rich in unsaturated fatty acids, which are also essential in the human body [36]; and the effects of unsaturated fatty acids are currently under investigation [37]. The dietary substitution of MFA or n-6 PFAs for SFA lowers blood cholesterol and may have beneficial effects on inflammation, thrombosis, and vascular reactivity [38-41]. The PFA content was measured in the 45 walnut samples from the five provinces, as shown in Figure 5(b). The PFA content was higher than $50 \%$ in 43 walnuts from the five provinces, expect Yunnan-Dabaike and ShuangjiangYangpao, indicating that walnut kernels were a good source of PFAs. The average PFA contents were similar in walnuts from Hebei and Shaanxi $(62.70 \pm 2.02 \%$ and $61.91 \pm 3.63 \%$, respectively). The average PFA content was around $57 \%$ in Shandong $(56.69 \pm 6.13 \%)$ and Sichuan $(57.19 \pm 0.38 \%)$, which was a little lower than that in Hebei and Shaanxi. The PFA content was also $57.50 \pm 4.27 \%$ in Yunnan province, but the content ranged from $45.62 \%$ to $64.88 \%$.

The MFA such as oleic acid content was high in pecan and less susceptible to lipid peroxidation; hence, it might have an advantage over PFAs [42]. Walnut was reported as a good source of MFAs [43]. Hence, the MFA content was measured in the 45 walnut samples, as shown in Figure 5(c). The average contents of
MFA content in walnuts from the Qinling-Daba Mountains and northeastern China along the Yellow River basin, including Shaanxi, Sichuan, Hebei, and Shandong, were $29.07 \pm 4.05 \%$, $34.20 \pm 1.92 \%, 29.3 \pm 4.04 \%$, and $35.24 \pm 7.09 \%$, respectively. In Yunnan, the average MFA content was $34.89 \pm 4.27 \%$, with a wide range of $27.50 \%-46.11 \%$.

3.5. Saturated Fatty Acids. Stearic acid is one of the main forms of SFAs in nuts. The content of stearic acid was next measured in the 45 walnut samples, as shown in Figure 6(a). The average steric acid content was higher than $2.5 \%$ in walnuts from Hebei, Shandong, Shaanxi, and Sichuan (from $2.9 \%$ to $4.1 \%)$. However, the average stearic acid content in walnuts from Yunnan was $2.44 \pm 0.48 \%$, which was much lower than that in walnuts from the other four provinces. Also, the stearic acid content in walnuts from Yunnan ranged from $1.70 \%$ to $3.75 \%$. Oleic acid is the main MFA component, and walnut genotypes have been described with high oleic acid content [44]. The present study next measured oleic acid content in walnuts from the five main walnut-producing provinces, as shown in Figure 6(b). Similar to what was observed for stearic acid, walnuts from Hebei and Shaanxi showed a similar oleic acid content ( $28.48 \pm 4.02 \%$ and $27.19 \pm 2.35 \%$, respectively), but walnuts from Shandong (34.22 $\pm 7.07 \%)$ and Sichuan $(33.24 \pm 1.92 \%)$ had slightly higher oleic acid content. Walnuts from Yunnan showed a similar average oleic acid content to those from Hebei and Shaanxi. However, the content distribution of oleic acid in walnuts from Yunnan was pretty large, from 


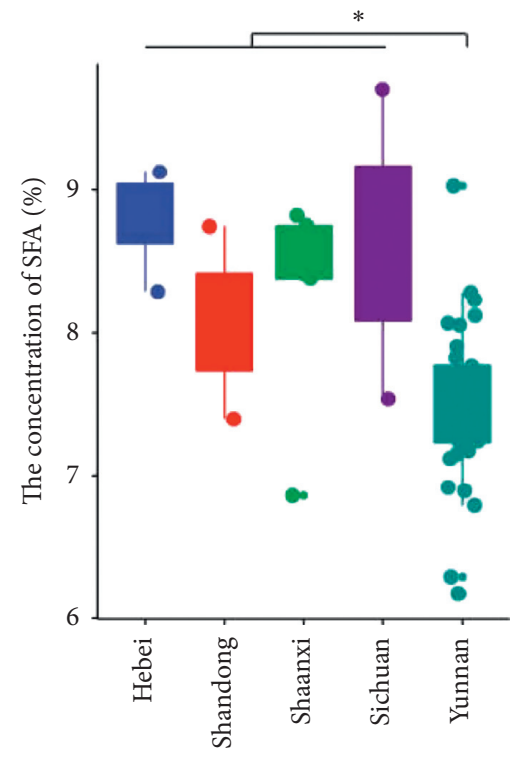

Five provinces in China

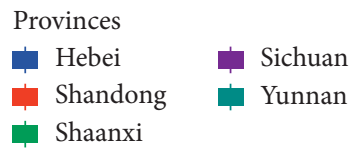

(a)

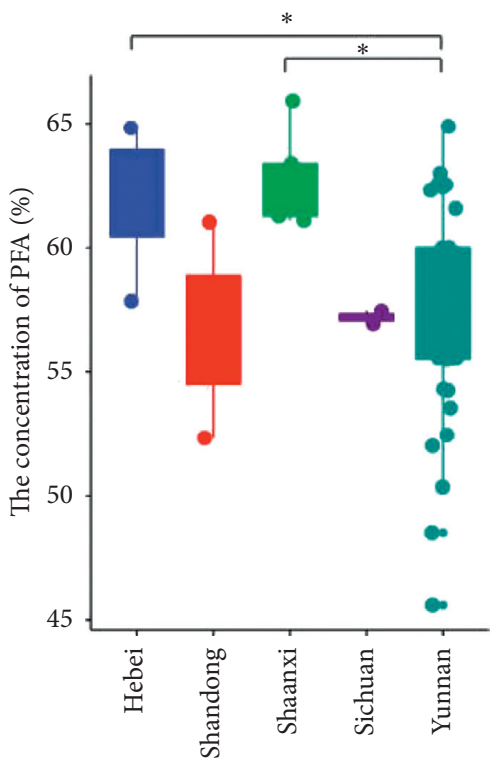

Five provinces in China Provinces

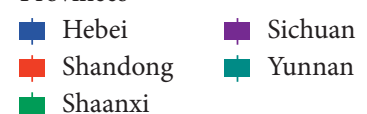

(b)

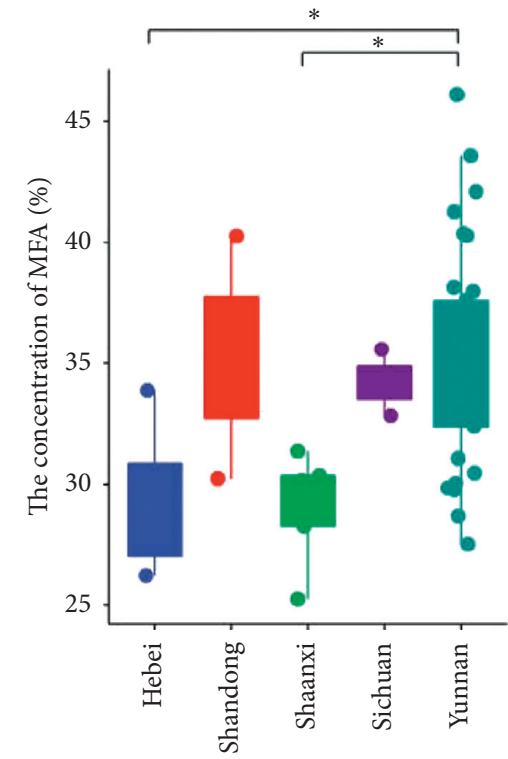

Five provinces in China Provinces

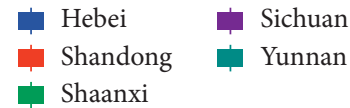

(c)

FIgURE 5: The (a) saturated fatty acid, (b) polyunsaturated fatty acids, and (c) monounsaturated fatty acid in 45 different types of walnut kernels from the main five walnut-planting provinces in China. Dunnett's multiple-comparison tests were implemented for statistical analysis. Raw $p$ value was labeled, ${ }^{*} p<0.1$ and ${ }^{* *} p<0.05$.

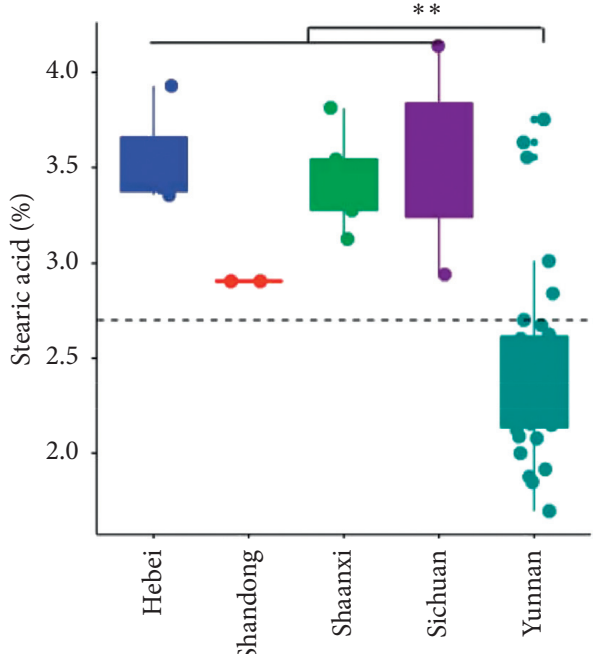

Five provinces in China

Provinces

Hebei

Shandong

Shaanxi

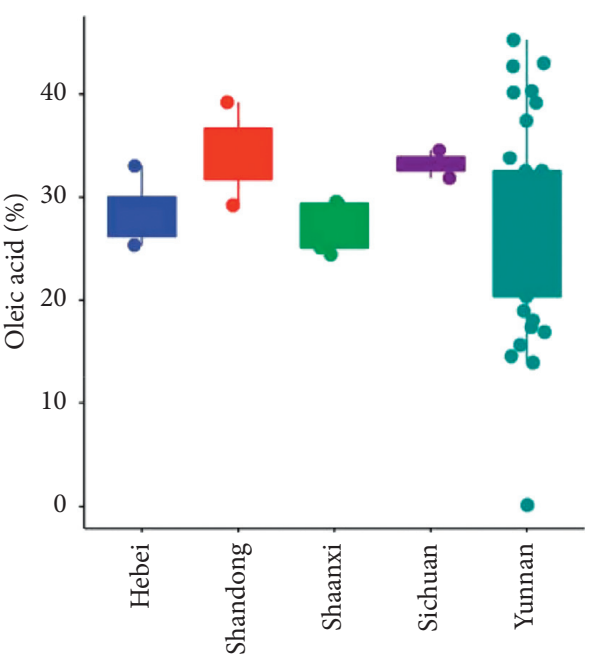

Five provinces in China

Provinces

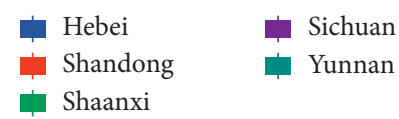

(b)

FIgURE 6: Continued. 


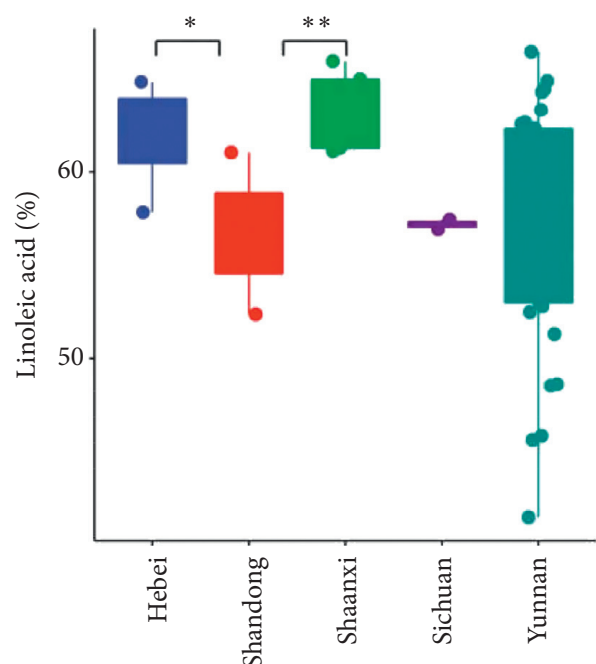

Five provinces in China

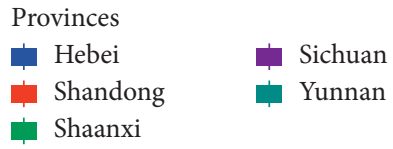

(c)
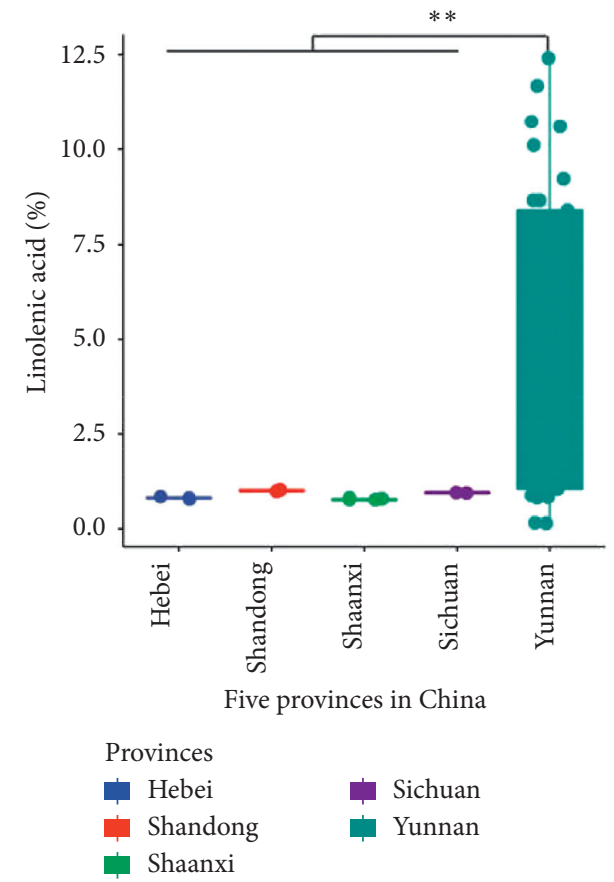

(d)

Figure 6: The (a) stearic acid, (b) oleic acid, (c) linoleic acid, and (d) linolenic acid in 45 different types of walnut kernels from the main five walnut-planting provinces in China. Dunnett's multiple-comparison tests were implemented for statistical analysis. Raw $p$ value was labeled, ${ }^{*} p<0.05$ and ${ }^{* *} p<0.01$.

$12.9 \%$ to $45.3 \%$, which might be attributed to the different varieties.

Linoleic acid, an $n-3$ PFA of plant origin, can reduce blood cholesterol and prevent atherosclerosis. Walnut kernels contain higher amounts of plant-derived $\alpha$-linoleic acid compared to other nut types $[45,46]$. Hence, the linoleic acid content was measured in the 45 walnut samples, as shown in Figure 6(c). The average linoleic acid content was higher than $50.0 \%$ in walnuts from Hebei, Shandong, Shaanxi, and Sichuan. The average linoleic acid content showed an opposite trend to the oleic acid content. The $\alpha$-linoleic acid contents were pretty similar in walnuts from Hebei and Shaanxi $(61.91 \pm 3.63 \%$ and $63.33 \pm 2.16 \%$, respectively), which were a little bit higher than those in walnuts from Shandong and Sichuan. $\alpha$-linolenic acid is an essential fatty acid in the human diet and is the principal $n-3$ PFA in the western diet [47]. The $\alpha$-linolenic acid content in the 45 walnut samples from the major five walnut-producing provinces was also tested, as shown in Figure 6(d). The average $\alpha$-linolenic acid content in walnuts from Yunnan was $5.24 \%$, which was higher than that in walnuts from the other provinces (average content was $0.8 \%$ ).

3.6. Amino Acids. The amino acid composition of walnut can reflect the protein content or the proportion of walnut protein and its amino acid composition. The PCA score illustrated that the majority of species of walnuts from Yunnan differed from those in the other provinces. The overlap components were species introduced from other provinces, as shown in Figure $7(\mathrm{a})$. The amino acid compositions were similar for walnuts from Hebei, Shandong, and Sichuan. Shaanxi has unique geographical and climatic characteristics. The amino acids composition in Shaanxi displayed a different distribution in the PCA score. Combined with the PCA loading analysis, the main amino acids of arginine and lysine were abundant in walnuts. Walnuts also contain a precursor of nitric oxide, a potent vasodilator that can inhibit platelet adhesion and aggregation. Due to the lower lysine/arginine ratio in plant proteins, the arginine from plant sources has high bioavailability [48]. Walnuts from Yunnan have higher contents of methionine and proline. The remaining amino acids show the opposite trends, as shown in Figure 7(b).

Heat-map analysis was performed to construct an overview of active ingredients in the tested walnut samples. "Euclidean" clustering was applied for analysis of the results for walnuts from the five provinces to understand potential regional differences better. Most Yunnan walnuts were clustered in the analysis. Walnuts from Hebei, Shandong, Sichuan, and Shaanxi mostly contained the same ingredients. The clustering analysis of the 31 active ingredients revealed clustering of the amino acids except proline and methionine, which were richer in walnuts from Yunnan. Macroscopically, lower levels of amino acids were found in walnuts from Yunnan, making these walnuts less rich in crude proteins. The contents of PFA, linoleic acid, FA, and stearic acid were also lower in walnuts from Yunnan compared with walnuts from the other four provinces, as shown in Figure 8. However, walnuts from Yunnan were richer in flavonoids, vitamin E, crude fat, and linolenic acid. 

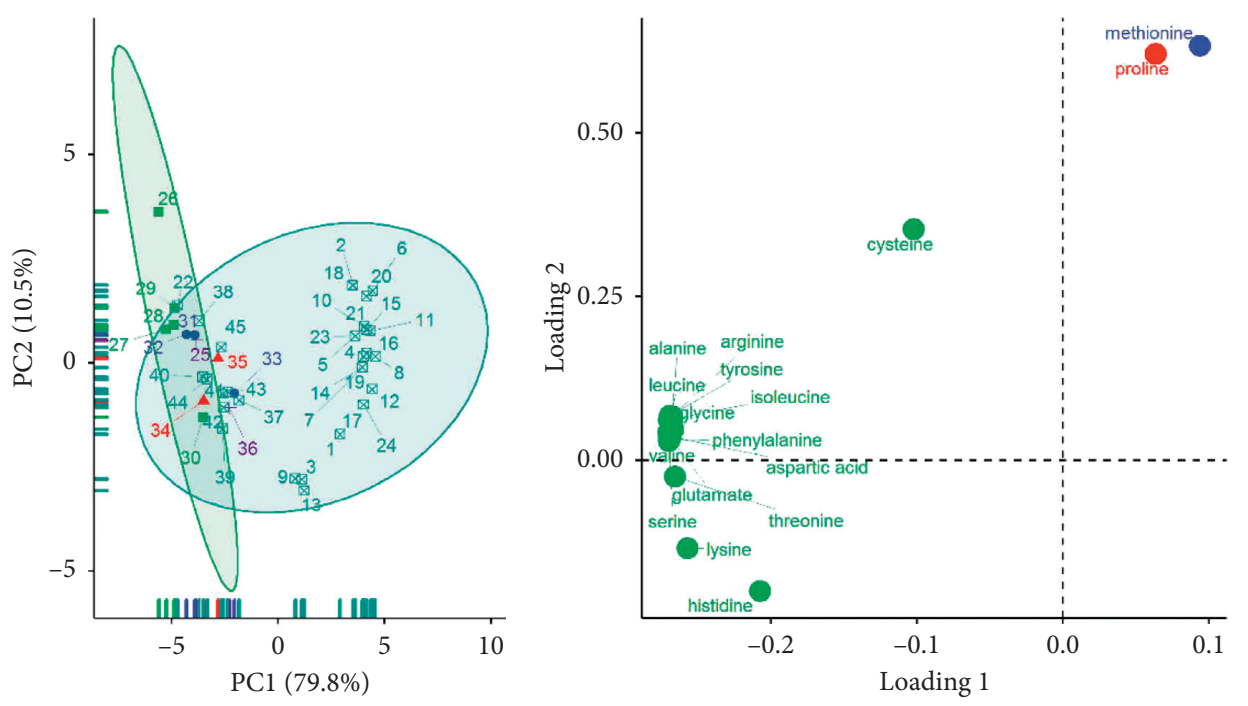

\begin{tabular}{|c|c|}
\hline Provinces & \\
\hline$\boxplus$ Hebei & - Sichuan \\
\hline Shandong & 母 Yunnan \\
\hline
\end{tabular}

(a)

(b)

FIgURE 7: (a) PCA score of 45 different types of walnut kernels from the main five walnut-planting provinces in China. (b) PCA loading of the 17 amino acids in 45 different types of walnut kernels. The first three significant contributed amino acids were labeled with colors.

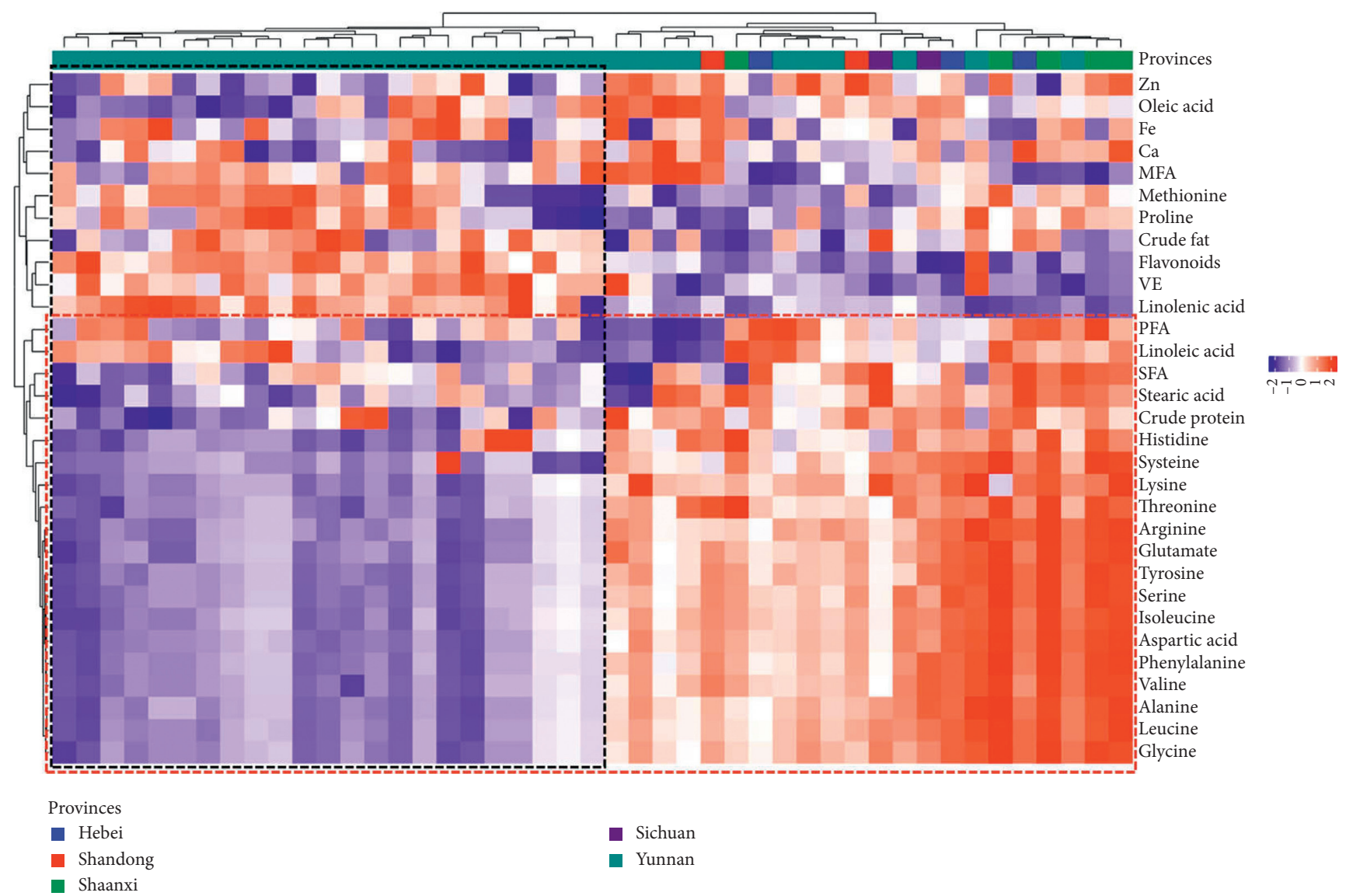

FIGURE 8: The heat map of the main physical and chemical factors in 45 different types of walnuts from the main five walnut-planting provinces in China. 
No significant differences were found in trace elements, including zinc, iron, and calcium, in the walnuts from different provinces.

\section{Conclusions}

The present study determined the main nutritional components and species differences of 45 walnut samples from different areas of China, including Shaanxi, Hebei, Shandong, Sichuan, and Yunnan. The results showed variation in composition and content in the analyzed walnut samples in different growing environments, with differences in the contents of flavonoids, vitamin E, trace elements, fatty acids, and amino acids. Differences were observed between walnut samples from different planting areas. PCA score and heatmap results illustrated that walnuts from Yunnan were different from those from the other provinces, possibly due to variations in the growing environment or genetic diversity. The data obtained in the present study provided a better understanding of the differences in the composition of different walnut species from the main growing areas in China. The results might facilitate the protection of germplasm resources, guide walnut processing, and enhance the qualities of walnut products.

\section{Data Availability}

No data were used to support this study.

\section{Conflicts of Interest}

The authors declare that they have no conflicts of interest.

\section{Acknowledgments}

This work was supported by the Yunnan Province Science and Technology Plan (2018ZG003).

\section{References}

[1] C. W. C. Kendall, A. Esfahani, A. R. Josse, L. S. A. Augustin, E. Vidgen, and D. J. A. Jenkins, "The glycemic effect of nutenriched meals in healthy and diabetic subjects," Nutrition, Metabolism and Cardiovascular Diseases, vol. 21, pp. S34-S39, 2011.

[2] P. Pollegioni, K. Woeste, F. Chioccine et al., "Rethinking the history of common walnut (Juglans regia L.) in Europe: its origins and human interactions," PLoS ONE, vol. 12, pp. 1-24, 2017.

[3] X. Fan, K. D. Hyde, M. Liu, Y. Liang, and C. Tian, "Cytospora species associated with walnut canker disease in China, with description of a new species C. gigalocus," Fungal Biology, vol. 119, no. 5, pp. 310-319, 2015.

[4] G. Li, F. Liu, J. Li, Q. Liu, and S. Chen, "Characterization of Botryosphaeria dothidea and Lasiodiplodia pseudotheobromae from English walnut in China," Journal of Phytopathology, vol. 164, no. 5, pp. 348-353, 2016.

[5] H. Han, K. E. Woeste, Y. Hu et al., "Genetic diversity and population structure of common walnut (Juglans regia) in China based on EST-SSRs and the nuclear gene phenylalanine ammonia-lyase (PAL)," Tree Genetics and Genomes, vol. 12, p. 111, 2016.
[6] L. Feng, F. Peng, X. Wang, M. Li, H. Lei, and H. Xu, "Identification and characterization of antioxidative peptides derived from simulated in vitro gastrointestinal digestion of walnut meal proteins," Food Research International, vol. 116, pp. 518-526, 2018.

[7] X. Rong-Ting, "Discussion on the origin of walnut in China," I International Symposium on Walnut Production, vol. 284, pp. 353-362, 1989.

[8] S. J. Dai, J. X. Qi, C. R. Duan et al., "Abnormal development of pollen and embryo sacs contributes to poor fruit set in walnut (Juglans hopeiensis)," The Journal of Horticultural Science and Biotechnology, vol. 89, no. 3, pp. 273-278, 2014.

[9] J. Tian, Y. Wu, Y. Wang, and F. Han, "Development and prospects of the walnut industry in China," VI International Walnut Symposium, vol. 861, pp. 31-38, 2009.

[10] G. Wu, Y. Wang, Q. Wang, N. Ding, and C. Ren, "Special germplasm resources of cultivated walnut in China," Acta Horticulturae, vol. 1050, pp. 49-53, 2014.

[11] X. Xu, H. Zhang, J. Yue, T. Xie, Y. Xu, and Y. Tian, "Predicting shifts in the suitable climatic distribution of walnut (Juglans regia L.) in China: maximum entropy model paves the way to forest management," Forests, vol. 9, p. 103, 2018.

[12] J. Li, P.-S. Xu, Z.-X. Zou et al., "Three new compounds from the roots of Juglans mandshurica Maxim," Phytochemistry Letters, vol. 20, pp. 40-44, 2017.

[13] L. V. M. Assis, J. S. S. Pinto, and F. M. Lancas, "Comparison among different extraction methods (PFE, SFE, Sonication, Soxhlet) for the isolation of organic compounds from coal," Journal of Microcolumn Separations, vol. 12, no. 5, pp. 292301, 2000.

[14] J. Salimon, T. A. Omar, and N. Salih, "Comparison of two derivatization methods for the analysis of fatty acids and transfatty acids in bakery products using gas chromatography," The Scientific World Journal, vol. 2014, Article ID 906407, 10 pages, 2014.

[15] F. Zhang, H. Cai, X. Liu et al., "Simultaneous determination of 19 fatty acids in Antrodia camphorata by derivatized GC-MS and evaluation of antioxidant activity of Antrodia camphorate crude oil," Archives of Pharmacal Research, vol. 1, 2015.

[16] X. Li, W. Kong, W. Shi, and Q. Shen, "A combination of chemometrics methods and GC-MS for the classification of edible vegetable oils," Chemometrics and Intelligent Laboratory Systems, vol. 155, pp. 145-150, 2016.

[17] A. Marcó, R. Rubio, R. Compano, R. Compano, and I. Casals, "Comparison of the Kjeldahl method and a combustion method for total nitrogen determination in animal feed," Talanta, vol. 57, no. 5, pp. 1019-1026, 2002.

[18] X. Xiong, Y. Yin, Y. Huang et al., "Methods of amino acid analysis," Nutritional and Physiological Functions of Amino Acids in Pigs, Springer, Berlin, Germany, pp. 217-229, 2013.

[19] H. Zhu, Y. Wang, Y. Liu, Y. Xia, and T. Tang, "Analysis of flavonoids in Portulaca oleracea L. by UV-Vis spectrophotometry with comparative study on different extraction technologies," Food Analytical Methods, vol. 3, no. 2, pp. 90-97, 2010.

[20] M. Ryynänen, A.-M. Lampi, P. Salo-Väänänen, V. Ollilainen, and V. Piironen, "A small-scale sample preparation method with HPLC analysis for determination of tocopherols and tocotrienols in cereals," Journal of Food Composition and Analysis, vol. 17, no. 6, pp. 749-765, 2004.

[21] J. E. Tahan, J. M. Sanchez, V. A. Granadillo, H. S. Cubillan, and R. A. Romero, "Concentration of total $\mathrm{Al}, \mathrm{Cr}, \mathrm{Cu}, \mathrm{Fe}, \mathrm{Hg}$, $\mathrm{Na}, \mathrm{Pb}$, and $\mathrm{Zn}$ in commercial canned seafood determined by atomic spectrometric means after mineralization by 
microwave heating," Journal of Agricultural and Food Chemistry, vol. 43, no. 4, pp. 910-915, 1995.

[22] A. Solar, M. Colarič, V. Usenik, and F. Stampar, "Seasonal variations of selected flavonoids, phenolic acids and quinones in annual shoots of common walnut (Juglans regia L.)," Plant Science, vol. 170, no. 3, pp. 453-461, 2006.

[23] J. S. Amaral, M. R. Alves, R. M. Seabra, and B. P. P. Oliveira, "Vitamin E composition of walnuts (Juglans regia L.): a 3-year comparative study of different cultivars," Journal of Agricultural and Food Chemistry, vol. 53, no. 13, pp. 5467-5472, 2005.

[24] J. Nriagu, "Zinc deficiency in human health," Encyclopedia of Environmental Health, vol. 34, pp. 789-800, 2011.

[25] T. Fukada, S. Yamasaki, K. Nishida, M. Murakami, and T. Hirano, "Zinc homeostasis and signaling in health and diseases," JBIC Journal of Biological Inorganic Chemistry, vol. 16, no. 7, pp. 1123-1134, 2011.

[26] S. Hojyo and T. Fukada, "Zinc transporters and signaling in physiology and pathogenesis," Archives of Biochemistry and Biophysics, vol. 611, pp. 43-50, 2016.

[27] C. M. Chaparro, "Setting the stage for child health and development: prevention of iron deficiency in early infancy," The Journal of Nutrition, vol. 138, no. 12, pp. 2529-2533, 2008.

[28] S. J. Cronin, C. J. Woolf, G. Weiss, and J. M. Penninger, "The role of iron regulation in immunometabolism and immunerelated disease," Frontiers in Molecular Biosciences, vol. 6, p. 116, 2019.

[29] S. Maggini, A. Pierre, and P. Calder, "Immune function and micronutrient requirements change over the life course," Nutrients, vol. 10, no. 10, p. 1531, 2018.

[30] K. Corbeels, L. Verlinden, M. Lannoo et al., "Thin bones: vitamin $\mathrm{D}$ and calcium handling after bariatric surgery," Bone Reports, vol. 8, pp. 57-63, 2018.

[31] E. Pchitskaya, E. Popugaeva, and I. Bezprozvanny, "Calcium signaling and molecular mechanisms underlying neurodegenerative diseases," Cell Calcium, vol. 70, pp. 87-94, 2018.

[32] I. J. Cindric, M. Zeiner, and D. Hlebec, "Mineral composition of elements in walnuts and walnut oils," International Journal of Environment Research and Public Health, vol. 15, p. 2674, 2018.

[33] M. I. Tapia, J. R. Sánchez-Morgado, J. García-Parra, R. Ramírez, T. Hernández, and D. González-Gómez, "Comparative study of the nutritional and bioactive compounds content of four walnut (Juglans regia L.) cultivars," Journal of Food Composition and Analysis, vol. 31, no. 2, pp. 232-237, 2013.

[34] X. Feng, H. Zhou, S. Zulfiqar et al., "The phytogeographic history of common walnut in China," Frontiers in Plant Science, vol. 9, p. 1399, 2018.

[35] Q. Li, R. Yin, Q. Zhang et al., "Chemometrics analysis on the content of fatty acid composition of different walnut (Juglans regia L.) varieties," Chinese Agriculture Science Bulletin, vol. 243, pp. 141-149, 2017.

[36] E. Sánchez-Zapata, J. Díaz-Vela, M. L. Pérez-Chabela, J. A. Pérez-Alvarez, and J. Fernández-López, "Evaluation of the effect of tiger nut fibre as a carrier of unsaturated fatty acids rich oil on the quality of dry-cured sausages," Food and Bioprocess Technology, vol. 6, no. 5, pp. 1181-1190, 2013.

[37] U. N. Das, "Arachidonic acid and other unsaturated fatty acids and some of their metabolites function as endogenous antimicrobial molecules: a review," Journal of Advanced Research, vol. 11, pp. 57-66, 2018.
[38] P. An, S. Qi, J. E. Manson, W. C. Willett, and F. B. Hi, "Walnut consumption is associated with lower risk of type 2 diabetes in women," The Journal of Nutrition, vol. 143, pp. 512-518, 2013.

[39] Y. Kara, A. Kocak, O. B. Citil, and E. Tulukcu, "A comparative study of the fatty acid composition and lipid content of Salvia sclarea," Chemistry of Natural Compounds, vol. 46, no. 4, pp. 612-614, 2010.

[40] M. Khodarahmi and L. Azadbakht, "The association between different kinds of fat intake and breast cancer risk in women," International Journal of Preventive Medicine, vol. 5, pp. 6-15, 2014.

[41] E. Ros and J. Mataix, "Fatty acid composition of nutsimplications for cardiovascular health," British Journal of Nutrition, vol. 96, no. S2, pp. S29-S35, 2006.

[42] J. E. Villarreall-Lozoya, L. Lombardini, and L. CisnerosZevallos, "Electron-beam irradiation effects on phytochemical constituents and antioxidant capacity of pecan kernels [Carya illinoinensis (Wangenh) K. Koch] during storage," Journal of Agricultural and Food Chemistry, vol. 57, no. 22, pp. 1073210739, 2009.

[43] I. Masako, K. Misaki, K. Daisuke, T. Hiroke, S. Masao, and I. Katsumi, "Differential effect of walnut oil and safflower oil on the serum cholesterol level and lesion area in the aortic root of apolipoprotein E-deficient mice," Bioscience Biotechnology and Biochemistry, vol. 66, pp. 141-146, 2014.

[44] A. Rabadán, J. E. Pardo, R. Gómez, and M. ÁVAREZ--OrtR, "Evaluation of physical parameters of walnut and walnut products obtained by cold pressing," LWT, vol. 91, pp. 308314, 2018.

[45] S. R. Ahemd, A. Shafique, F. Azeem et al., "Edible oil," in Green Sustainable Process for Chemical and Environmental Engineering and Science, pp. 99-126, Elsevier, Amsterdam, Netherlands, 2021.

[46] S. Morgillo, A. M. Hill, and A. M. Coates, "The effects of nut consumption on vascular function," Nutrients, vol. 11, no. 1, p. 116, 2019.

[47] G. C. Burdge, "Metabolism of $\alpha$-linolenic acid in humans," Prostaglandins, Leukotrienes and Essential Fatty Acids, vol. 75, no. 3, pp. 161-168, 2006.

[48] D. Kritchevsky, S. A. Tepper, S. K. Czarnecki, and D. M. Klurfeld, "Atherogenicity of animal and vegetable protein,” Atherosclerosis, vol. 41, no. 2-3, pp. 429-431, 1982. 